\title{
HOMOMORPHISMS ON GROUPS AND INDUCED MAPS ON CERTAIN ALGEBRAS OF MEASURES( ${ }^{1}$ )
}

\author{
BY \\ CHARLES F. DUNKL AND DONALD E. RAMIREZ
}

\begin{abstract}
Suppose that $\varphi$ is a continuous homomorphism of a locally compact group $G$ into another such group, $H$, then $\varphi$ induces in a natural way a homomorphism $\varphi^{*}$ of the measure algebra of $G$, called $M(G)$, into $M(H)$. The action of $\varphi^{*}$ on the subspace $M_{0}(G)$ is studied in this paper. The space $M_{0}(G)$ is the nonabelian analogue to the space of measures on a locally compact abelian group whose Fourier-Stieltjes transforms vanish at infinity, and is defined herein. We prove that if $\varphi$ is an open homomorphism then $\varphi^{*}\left(M_{0}(G)\right) \subset M_{0}(H)$. If $G$ and $H$ are abelian and $\varphi$ is not open, then $\varphi^{*}(M(G)) \cap M_{0}(H)=\{0\}$. The main tool for this theorem is the fact, proved herein, that $\varphi$ is open if and only if its adjoint, $\hat{\varphi}: \hat{H} \rightarrow \hat{G}$, is proper (where $\hat{G}, \hat{H}$ are the character groups of $G, H$ resp.). Further properties of $M_{0}(G)$ for abelian or compact groups $G$ are derived.
\end{abstract}

Introduction. Suppose that $\varphi$ is a continuous homomorphism of a locally compact group $G$ into another such group, $H$, then $\varphi$ induces in a natural way a homomorphism $\varphi^{*}$ of the measure algebra of $G$, called $M(G)$, into $M(H)$. The action of $\varphi^{*}$ on the subspaces $L^{1}(G)$ (measures absolutely continuous with respect to left Haar measure of $G)$ and $M_{0}(G)$ is studied in this paper. The space $M_{0}(G)$ is the nonabelian analogue to the space of measures on a locally compact abelian group whose Fourier-Stieltjes transforms vanish at infinity, and is defined herein. This space was originally defined and studied in a previous paper of the authors. A theorem in $\S 2$ says that if $\varphi$ is an open homomorphism then $\varphi^{*}\left(M_{0}(G)\right) \subset M_{0}(H)$. If $G$ and $H$ are abelian, an analysis of the topological properties of $\varphi$ and $\hat{\varphi}$, the induced map of $\hat{H} \rightarrow \hat{G}$ (where $\hat{G}$ is the character group of $G$ ), carried out in $\S 1$, gives a converse to the above theorem; in fact, if $\varphi$ is not open, then $\varphi^{*}(M(G))$ $\cap M_{0}(H)=\{0\}$. The main tool for this theorem is the fact, proved in $\S 1$, that $\varphi$ is open if and only if $\hat{\varphi}$ is proper (i.e., the inverse image under $\hat{\varphi}$ of a compact set is compact). In $\S 3$ further properties of $M_{0}(G)$ for abelian or compact groups $G$ are derived. One result is an extension of a classical result of Rajchman to abelian groups with a certain property, and examples are presented to illustrate this property. Another result says that if $\varphi$ is an ergodic homomorphism of a compact

Received by the editors January 23, 1970 and, in revised form, June 6, 1970.

AMS 1968 subject classifications. Primary 2265, 4250, 4256, 4680.

Key words and phrases. Proper homomorphism, open homomorphism, measure algebra, translation of measures is continuous, translation invariant norm on measures, FourierStieltjes transforms, Fourier-Stieltjes transforms vanishing at infinity, local direct product of groups, ergodic homomorphism, band of measures.

(1) This research was supported in part by NSF contract number GP 8981.

Copyright (C) 1971, American Mathematical Society 
group $G$ into itself and $\mu \in M_{0}(G)$, then $\left(\varphi^{*}\right)^{n} \mu$ converges to $\mu(G)$ times the Haar measure of $G$ as $n \rightarrow \infty$, in the weak-* topology.

1. Topological properties of homomorphisms. In this paper, $G$ and $H$ will always denote locally compact groups with the identity element of each denoted by $e$, and $\varphi$ will denote a continuous homomorphism of $G$ into $H$. Below we give definitions of further properties that $\varphi$ may have.

Definition. (a) $\varphi$ is said to be proper if $\varphi^{-1}(K)$ is compact in $G$ whenever $K$ is a compact subset of $H$.

(b) $\varphi$ is said to be open [resp. closed] if $\varphi(U)$ is open [resp. closed] in $H$ whenever $U$ is open [resp. closed] in $G$.

(c) $\varphi$ is said to be a strict morphism if $\varphi(G)$ with the relative topology induced by $H$ is homeomorphic to $G / \operatorname{ker} \varphi$.

REMARK. It is well known that $\varphi$ is a strict morphism if and only if $\varphi$ is open onto $\varphi(G)$ with the relative topology $[1$, p. 236]. Further, if $\varphi$ is a strict morphism then $\varphi(G)$ is a closed subgroup of $H$, since it is locally compact in the relative topology. We state now some easy consequences of the definition of proper maps, with short or omitted proofs. Some will be in terms of generalized sequences, or nets.

1. Proposition. $\varphi$ is proper if and only if $x_{\alpha} \rightarrow \infty$ in $G$ implies $\varphi x_{\alpha} \rightarrow \infty$ in $H$, for all nets $\left\{x_{\alpha}\right\} \subset G$.

The following uses the group properties to give a local characterization of proper homomorphisms.

2. Proposition. $\varphi$ is proper if and only if for any compact neighborhood $V$ of $e$ in $H, \varphi^{-1}(V)$ is a compact neighborhood of $\operatorname{ker} \varphi$, the kernel of $\varphi$.

The following describes the possible limit points of nets $\left\{\varphi x_{\alpha}\right\}$ where $x_{\alpha} \rightarrow \infty$ in $G$.

3. Proposition. If $\varphi$ is not proper, then for any $y \in \mathrm{cl}(\varphi(G))$, there exists a net $\left\{x_{\alpha}\right\} \subset G$ such that $x_{\alpha} \rightarrow \infty$ and $\varphi x_{\alpha} \rightarrow y$. Equivalently, if $\varphi$ is not proper, then

$$
\bigcap\left\{\mathrm{cl}\left(\varphi\left(K^{c}\right)\right): K \text { compact } \subset G\right\}=\operatorname{cl}(\varphi(G))
$$

where $K^{c}$ is the set-theoretic complement of $K$.

Proof. If $\varphi$ is not proper, then there is a compact set $E \subset H$, such that $\varphi^{-1}(E)$ is not compact in $G$. Let $\left\{x_{\alpha}\right\}$ be a net in $\varphi^{-1}(E)$ such that $x_{\alpha} \rightarrow \infty$. Extract a subnet $\left\{x_{\beta}\right\}$ such that $\varphi x_{\beta} \vec{\beta}>y$ for some $y \in E$. Then clearly $y \in \operatorname{cl}(\varphi(G))$, and further for any $x \in G, x x_{\beta} \rightarrow \infty$, and $\varphi\left(x x_{\beta}\right) \vec{\beta}(\varphi x) y$. So the set of limit points is invariant under translation by points in $\varphi(G)$, and it is also closed and nonempty; thus it equals $\mathrm{cl}(\varphi(G))$.

4. Proposition. $\varphi$ is proper if and only if $\varphi$ is a strict morphism and $\operatorname{ker} \varphi$ is compact. 
Proof. Consider the canonical decomposition

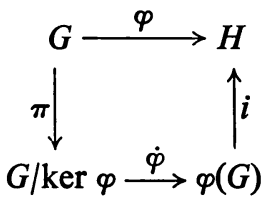

If $\varphi$ is proper, then $\varphi$ is a closed mapping and in particular $\varphi(G)$ is closed in $H$ $[1$, p. 101]. It follows that $\dot{\varphi}$ is a closed mapping and thus $\varphi$ is a strict morphism.

If $\varphi$ is a strict morphism and $K$ is a compact set in $H$, then $\dot{\varphi}^{-1}(K \cap \varphi(G))$ is compact in $G / \operatorname{ker} \varphi$. Now since $\operatorname{ker} \varphi$ is compact, $\varphi^{-1}(K)=\pi^{-1} \dot{\varphi}^{-1}(K \cap \varphi(G))$ is compact in $G$ [10, p. 109].

5. Corollary. If $H$ is discrete, then $\varphi$ is proper if and only if $\operatorname{ker} \varphi$ is compact.

6. Proposition. If $G$ is $\sigma$-compact, then $\varphi$ is a strict morphism if and only if $\varphi(G)$ is closed in $H$.

Proof. See [7, p. 42].

7. CoROllary. If $G$ is $\sigma$-compact, then $\varphi$ is proper if and only if $\varphi(G)$ is closed in $H$ and $\operatorname{ker} \varphi$ is compact.

NoTE. The remaining definition and theorems in this section pertain to (locally compact) abelian groups $G$ and $H$ with duals (i.e., character groups) $\hat{G}$ and $\hat{H}$ respectively.

Definition. For $\varphi$ a continuous homomorphism of $G$ into $H$, define the induced homomorphism $\hat{\varphi}$ of $\hat{H}$ into $\hat{G}$, by $(x, \hat{\varphi} \gamma)=(\varphi x, \gamma)$ for all $\gamma \in \hat{H}, x \in G$. Since the topologies of $\hat{G}$ and $\hat{H}$ can be taken to be the compact-open topologies as spaces of functions on $G$ and $H$, respectively, we see immediately that $\hat{\varphi}$ is a continuous homomorphism.

8. THEOREM. $\varphi$ is proper if and only if $\hat{\varphi}$ is open, and $\varphi$ is open if and only if $\hat{\varphi}$ is proper.

Proof. It is enough to prove half of this statement, since the map induced by $\hat{\varphi}$ on the character groups of $\hat{G}$ and $\hat{H}$ can be identified with $\varphi$ by the Pontryagin duality theorem. Suppose $\varphi$ is proper or open, then (see Proposition 4) $\varphi$ is a strict morphism and $\varphi(G)$ is a closed subgroup of $H$. We have the following:

$$
\begin{aligned}
& G \longrightarrow G / \operatorname{ker} \varphi \cong \varphi(G) \stackrel{i}{\longrightarrow} H \\
& \hat{G} \stackrel{i}{\longleftarrow}(\operatorname{ker} \varphi)^{\perp} \cong \hat{H} / \varphi(G)^{\perp} \longleftarrow \hat{H}
\end{aligned}
$$

where groups and maps in the same column are dual to each other. For if $E$ is a closed subgroup of $G$ and $E^{\perp}=\{\gamma \in \hat{G}:(x, \gamma)=1$ for all $x \in E\}$, then $E^{\perp}$ is the dual of $G / E$, and $E$ is the dual of $\hat{G} / E^{\perp}$ [14, Chapter 2]. Since $G / \operatorname{ker} \varphi$ is homeomorphic to $\varphi(G)$, we have that $\hat{H} / \varphi(G)^{\perp}$ is homeomorphic to $(\operatorname{ker} \varphi)^{\perp}$. Suppose 
that $\varphi$ is proper, then $\operatorname{ker} \varphi$ is compact, and to show that $\hat{\varphi}$ is open it is enough to show $i:(\operatorname{ker} \varphi)^{\perp} \rightarrow \hat{G}$ is open, that is, that $(\operatorname{ker} \varphi)^{\perp}$ is an open subgroup of $\hat{G}$. But $\operatorname{ker} \varphi$ is the dual of $\hat{G} /(\operatorname{ker} \varphi)^{\perp}$ and so $\hat{G} /(\operatorname{ker} \varphi)^{\perp}$ is discrete.

Now suppose $\varphi$ is open, then $\varphi(G)$ is an open subgroup of $H$, and ker $\hat{\varphi}=\varphi(G)^{\perp}$ which is compact, being the dual of $H / \varphi(G)$, so by Proposition $4, \hat{\varphi}$ is proper.

This theorem yields an easy proof of the following classical fact.

9. COROLLARY. If $G$ is a compact abelian group, then $G$ is divisible if and only if $G$ is connected.

Proof. $G$ is connected if and only if $\hat{G}$ has no torsion. If $\hat{G}$ has no torsion, then for each $n=2,3,4, \ldots$ the map $T_{n}: \gamma \mapsto n \gamma$ (additive notation) is one-to-one on $\hat{G}$ and thus proper, and so the induced map $\hat{T}_{n}: x \mapsto n x$ is open on $G$, so its image is an open subgroup of $G$ and thus $G$ itself. Similarly one can prove the converse.

2. Induced homomorphisms of the measure algebra. Here $G$ and $H$ will again denote locally compact groups with a continuous homomorphism $\varphi$ of $G \rightarrow H$, and $M(G)$ is the Banach algebra of finite regular Borel measures on $G$, with convolution implicitly defined by

$$
\int_{G} f d \mu * \nu=\int_{G} \int_{G} f(x y) d \mu(x) d \nu(y),
$$

for $\mu, \nu \in M(G)$ and $f \in C_{0}(G)$ (the space of continuous functions vanishing at infinity). $M(H)$ is of course similarly defined. Observe that the above convolution formula holds for bounded continuous functions $f$ on $G$.

Definition. Given $\varphi$, define the induced map $\varphi^{*}: M(G) \rightarrow M(H)$ by

$$
\int_{H} f d \varphi^{*} \mu=\int_{G} f(\varphi x) d \mu(x),
$$

for all $f \in C_{0}(H)$. Clearly $\varphi^{*}$ is linear and continuous with $\left\|\varphi^{*} \mu\right\| \leqq\|\mu\|$. By standard limit arguments we can see that $\int_{H} f d \varphi^{*} \mu=\int_{G} f(\varphi x) d \mu(x)$ for all bounded continuous functions $f$ on $H$.

10. Proposition. $\varphi^{*}$ is a continuous Banach algebra homomorphism of $M(G)$ into $M(H)$.

Proof. It is enough to show $\varphi^{*}(\mu * \nu)=\left(\varphi^{*} \mu\right) *\left(\phi^{*} \nu\right)$ for $\mu, \nu \in M(G)$. Let $f \in C_{0}(H)$ then

$$
\begin{aligned}
\int_{H} f d\left(\varphi^{*} \mu * \varphi^{*} \nu\right) & =\int_{H} \int_{H} f(s t) d \varphi^{*} \mu(s) d \varphi^{*} \nu(t) \\
& =\int_{G} \int_{G} f(\varphi x \varphi y) d \mu(x) d \nu(y) \quad \text { (by Fubini's theorem) } \\
& =\int_{G} \int_{G} f(\varphi(x y)) d \mu(x) d \nu(y)=\int_{G} f(\varphi x) d \mu * \nu(x) \\
& =\int_{H} f d \varphi^{*}(\mu * \nu) .
\end{aligned}
$$


Let $m_{G}$ and $m_{H}$ denote left invariant Haar measure on $G$ and $H$ respectively, and $L^{p}(G)$ is short for $L^{p}\left(G, m_{G}\right), 1 \leqq p<\infty$, and similarly $L^{p}(H)$. We identify $L^{1}(G)$ and $L^{1}(H)$ with closed ideals of $M(G)$ and $M(H)$ respectively in the standard way. (Now we introduce a lemma which will be used in three different cases.) For convenience we discuss a rather abstract situation in the following. Suppose $\mathscr{N}_{G}$ is a norm on $M(G)$, such that it is invariant under left translation (i.e. $\mathscr{N}_{G}(L(x) \mu)$ $=\mathscr{N}_{G}(\mu)$ where $L(x)$ is defined by $\int_{G} f d L(x) \mu=\int_{G} f(x y) d \mu(y)$, for all $\left.f \in C_{0}(G)\right)$, and $\mathscr{N}_{G}(\mu) \leqq\|\mu\|$ for all $\mu \in M(G)$. Let $X_{G}=\left\{\mu \in M(G): \mathscr{N}_{G}(L(x) \mu-\mu) \rightarrow 0\right.$ as $x \rightarrow e$ in $G$. Clearly $X_{G}$ is a closed subspace of $M(G)$, in fact if $\left\|\mu_{n}-\mu\right\| \rightarrow 0$, $\left\{\mu_{n}\right\} \subset X_{G}$, then $\mathscr{N}_{G}(L(x) \mu-\mu) \leqq 2\left\|\mu-\mu_{n}\right\|+\mathscr{N}_{G}\left(L(x) \mu_{n}-\mu_{n}\right)$, etc. Note that by the translation invariance the map $x \mapsto L(x) \mu$ is uniformly continuous on $G$ for each $\mu \in X_{G}$ where we give $M(G)$ the $\mathscr{N}_{G}$-topology. Suppose further that $\mathscr{N}_{H}$ is a norm on $M(H)$ with properties similar to $\mathscr{N}_{G}$, and let $X_{H}$ be similarly defined.

11. Lemma. Let $\mathscr{N}_{G}, \mathscr{N}_{H}, X_{G}, X_{H}$ be as above and suppose that $\varphi$ is open, and $\mathscr{N}_{H}\left(\varphi^{*} \mu\right) \leqq \mathscr{N}_{G}(\mu)$ for all $\mu \in M(G)$, then $\varphi^{*} X_{G} \subset X_{H}$.

Proof. Let $\mu \in X_{G}$ and $\varepsilon>0$, then there exists a neighborhood $U$ of $e$ in $G$ such that $x \in U$ implies $\mathscr{N}_{G}(L(x) \mu-\mu)<\varepsilon$. Since $\varphi$ is open we have that $\varphi(U)$ is a neighborhood of $e$ in $H$. Let $s \in \varphi(U)$, then there is $x \in U$ with $s=\varphi x$, and

$$
\mathscr{N}_{H}\left(L(s) \varphi^{*} \mu-\varphi^{*} \mu\right)=\mathscr{N}_{H}\left(\varphi^{*}(L(x) \mu-\mu)\right) \leqq \mathscr{N}_{G}(L(x) \mu-\mu)<\varepsilon
$$

(observe, for $f \in C_{0}(H)$, that $\int_{H} f d L(s) \varphi^{*} \mu=\int_{G} f(s \varphi y) d \mu(y)=\int_{G} f(\varphi(x y)) d \mu(y)$ $\left.=\int_{G} f(\varphi y) d L(x) \mu(y)\right)$.

We will now define some candidates for $\mathscr{N}_{G}, \mathscr{N}_{H}$. Let $\pi$ be a continuous unitary representation of $G$ on the Hilbert space $\mathscr{H}_{\pi}$, and define $\hat{\mu}(\pi)=\int_{G} \pi(x) d \mu(x)$, a weak integral giving a bounded operator on $\mathscr{H}_{\pi}$ with norm $\|\hat{\mu}(\pi)\|$. Let $N_{G}(\mu)$ $=\sup \{\|\hat{\mu}(\pi)\|: \pi$ is a continuous unitary representation of $G\}$. It is easy to see that $N_{G}$ satisfies the above hypotheses for $\mathscr{N}_{G}$, since for each $\pi,\left\|(L(x) \mu)^{\wedge}(\pi)\right\|=\|\hat{\mu}(\pi)\|$ $\leqq\|\mu\|$, and $N_{G}(\mu)=0$ implies $\mu=0$ by the Gel'fand Raikov theorem. Let $N_{G}^{\prime}(\mu)$ $=\|\hat{\mu}(\rho)\|$, where $\rho$ is the left regular representation of $G$ on $L^{2}(G)$, i.e. $\rho(x) f(y)$ $=f\left(x^{-1} y\right)$ for almost all $y \in G$. Thus $\hat{\mu}(\rho)$ is the convolution operator $f \mapsto \mu * f(y)$ $=\int_{G} f\left(x^{-1} y\right) d \mu(x)$ for almost all $y \in G, f \in L^{2}(G)$, and $N_{G}^{\prime}$ is its norm. Clearly $N_{G}^{\prime}$ also satisfies the above hypotheses for $\mathscr{N}_{G}$ and $N_{G}^{\prime}(\mu) \leqq N_{G}(\mu) \leqq\|\mu\|$.

Indeed we can obtain $N_{G}$ by taking the supremum over only the irreducible unitary continuous representations (see [5, §1.24]). In case the group $G$ is compact these representations are finite dimensional, and in fact $N_{G}(\mu)=N_{G}^{\prime}(\mu)$.

It is known $[5, \S 1.16]$ that $N_{G}(\mu)=N_{G}^{\prime}(\mu)$ for all $\mu \in M(G)$ if and only if $G$ is amenable (i.e. there exist invariant means on $L^{\infty}(G)$ ).

In a previous paper we showed that for the norms $\|\cdot\|$ (measure norm), $N_{G}$, and $N_{G}^{\prime}$ the corresponding space $X_{G}$ is in fact the closure of $L^{1}(G)$ in the pertinent norm [3]. Of course the fact that for $\|\cdot\|$ the space $X_{G}$ is $L^{1}(G)$ was discovered earlier by Rudin [13]. 
Definition. If $G$ is compact or abelian, then the space $X_{G}$ corresponding to $N_{G}=N_{G}^{\prime}$ is called $M_{0}(G)$, since, for $\mu \in M_{0}(G),\|\hat{\mu}(\pi)\| \rightarrow 0$ as $\pi \rightarrow \infty$ in the dual, or character group respectively. In fact, for $G$ compact, the dual $\hat{G}$ is a set of finite-dimensional representations. Following our notation in [4], we let $\mathscr{L}^{\infty}(\hat{G})$ $=\left\{\varphi=\left(\varphi_{\pi}\right)_{\pi \in \hat{G}}: \varphi_{\pi}\right.$ is a bounded operator on $\left.\mathscr{H}_{\pi},\|\varphi\|_{\infty}=\sup _{\pi}\left\|\varphi_{\pi}\right\|<\infty\right\}$, a $C^{*}$ algebra. Note that the Fourier transformation, $\mu \mapsto \hat{\mu}$, maps $M(G)$ one-to-one, into $\mathscr{L}^{\infty}(\hat{G})$. Further let $\mathscr{C}_{0}(\hat{G})=\left\{\varphi \in \mathscr{L}^{\infty}(\hat{G})\right.$ : for each $\varepsilon>0,\left\|\varphi_{\pi}\right\| \geqq \varepsilon$ for only finitely many $\pi \in \hat{G}\}$. This is clearly the closed subalgebra spanned by the elements of $\mathscr{L}^{\infty}(\hat{G})$ with finite support in $\hat{G}$ (which correspond to trigonometric polynomials on $G$ ). Thus $\hat{\mu} \in \mathscr{C}_{0}(\hat{G})$ if and only if $\mu$ can be approximated in $N_{G}$ norm (the norm in $\left.\mathscr{L}^{\infty}(\hat{G})\right)$ by elements of $L^{1}(G)$.

If $G$ is not amenable then we will denote the $N_{G}$-closure of $L^{1}(G)$ by $M_{0}(G)$, and the $N_{G}^{\prime}$-closure of $L^{1}(G)$ by $M_{0}^{\prime}(G)$. (This differs by a dash from the notation in [4].)

We showed in [4] that $M_{0}(G)$ and $M_{0}^{\prime}(G)$ are bands (a subspace $B$ of $M(G)$ is a band if $\mu \in B$ and $\nu$ absolutely continuous with respect to $\mu$ implies $\nu \in B$ ).

REMARK. We expect that there exist, in general, singular measures in $M_{0}(G)$. For example, every singular central measure on the $2 \times 2$ special unitary group $S U(2)$ not supported on ${ }^{ \pm}\left(\begin{array}{l}10 \\ 0\end{array}\right)$ is in $M_{0}(S U(2))$, and every singular zonal measure $\mu$ on the sphere $S^{n-1}=\left\{\xi \in R^{n}: \sum \xi_{i}^{2}=1\right\}(n \geqq 3)$ such that

$$
|\mu|\{(1,0, \ldots, 0),(-1,0, \ldots, 0)\}=0
$$

can be considered as a bi-invariant singular measure in $M_{0}(S O(n))$ where $S O(n)$ is the rotation group on $R^{n}$ and the isotropy subgroup is

$$
S O(n-1)=\{x \in S O(n):(1,0, \ldots, 0) x=(1,0, \ldots, 0)\}
$$

(see [2, p. 256] or $[3, \S 9.6 .16])$.

We now similarly define $N_{H}, N_{H}^{\prime}, M_{0}(H)$ and $M_{0}^{\prime}(H)$.

12. TheOREM. Let $\varphi$ be an open homomorphism of $G$ into $H$. Then $\varphi^{*}\left(L^{1}(G)\right)$ $\subset L^{1}(H)$ and $\varphi^{*}\left(M_{0}(G)\right) \subset M_{0}(H)$.

Proof. Taking $\mathscr{N}_{G}$ to be $\|\cdot\|$ in Lemma 11 we see immediately from the above remarks that $\varphi^{*}\left(L^{1}(G)\right) \subset L^{1}(H)$.

Let $\pi$ be a continuous unitary representation of $H$ and let $\mu \in M(G)$, then

$$
\left(\varphi^{*} \mu\right)^{\wedge}(\pi)=\int_{H} \pi(s) d \varphi^{*} \mu(s)=\int_{G} \pi(\varphi x) d \mu(x)=\hat{\mu}(\pi \circ \varphi) .
$$

We are here using the formula for $\varphi^{*} \mu$ for operator-valued functions, but this can be justified by weak-integral arguments. Now $\pi \circ \varphi$ is a continuous unitary representation of $G$ so

$$
\left\|\left(\varphi^{*} \mu\right)^{\wedge}(\pi)\right\|=\|\hat{\mu}(\pi \circ \varphi)\| \leqq N_{G}(\mu)
$$

thus $N_{H}\left(\varphi^{*} \mu\right) \leqq N_{G}(\mu)$. Now Lemma 11 shows that $\varphi^{*}\left(M_{0}(G)\right) \subset M_{0}(H)$. 
13. THEOREM. Let $\varphi$ be an open and proper homomorphism of $G$ into $H$, then $\varphi^{*}\left(M_{0}^{\prime}(G)\right) \subset M_{0}^{\prime}(H)$.

Proof. It is clearly enough to show that $N_{H}^{\prime}\left(\varphi^{*} \mu\right) \leqq N_{G}^{\prime}(\mu)$, that is $\left\|\left(\varphi^{*} \mu\right)^{\wedge}\left(\rho_{H}\right)\right\|$ $\leqq\left\|\hat{\mu}\left(\rho_{G}\right)\right\|$ for all $\mu \in M(G)$.

Suppose that $\nu \in M(H)$ and is supported by an open subgroup $E$ of $H$. Then it is clear that the left Haar measure of $H$ restricted to $E$ is a left Haar measure for $E$, and $L^{2}(H)$ is a direct sum of copies of $L^{2}(E)$ (one for each left coset $E s$ of $E$ ) and the convolution operator norm of $\nu$ acting on $L^{2}(H)$ is the same as that on $L^{2}(E)$.

Now let $\mu \in M(G)$, then $\varphi^{*} \mu$ is supported by $\varphi(G)$, an open subgroup of $H$, and furthermore, we can identify $\varphi(G)$ with $G / \operatorname{ker} \varphi$ (since $\varphi$ is a strict morphism). Thus we need only consider $\mu$ as a convolution operator on $L^{2}(G / \operatorname{ker} \varphi)$. Now $K=\operatorname{ker} \varphi$ is compact, and the Weil formula [15, pp. 42-45] says

$$
\int_{G / K} d m_{G / K}(K x) \int_{K} f(h x) d m_{K}(h)=\int_{G} f d m_{G} .
$$

If $f$ is constant on cosets of $K$, and $m_{K}$ is normalized to have $m_{K}(K)=1$ then $\int_{G / K} f(K x) d m_{G / K}(K x)=\int_{G} f d m_{G}$. Thus $L^{2}(G / \operatorname{ker} \varphi)$ can be identified with a closed subspace of $L^{2}(G)$, namely the space of functions in $L^{2}(G)$ which are constant on cosets of $\operatorname{ker} \varphi$, and further this subspace is invariant under translation and left convolution by measures. Thus if $\mu \in M(G), f \in L^{2}(G / K),\|\mu * f\|_{2} \leqq\|\hat{\mu}(\rho)\|\|f\|_{2}$, so the $L^{2}(G / K)$ operator norm for $\mu$ is dominated by $\|\hat{\mu}(\rho)\|$.

The following is a converse to Theorem 12 for the case of $G$ and $H$ being abelian. It says in fact that if for some $f \in L^{1}(G), \varphi^{*} f \in M_{0}(H)$ and $\varphi^{*} f \neq 0$, then $\varphi$ is open.

14. TheOREM. Suppose $G$ and $H$ are abelian, and $\varphi$ is not open. Then

$$
\varphi^{*}(M(G)) \cap M_{0}(H)=\{0\}
$$

in fact,

$$
\begin{aligned}
\lim \sup \left\{\left|\left(\varphi^{*} \mu\right)^{\wedge}(\gamma)\right|: \gamma \in \hat{H}, \gamma \rightarrow \infty\right\} & =\sup \left\{\left|\left(\varphi^{*} \mu\right)^{\wedge}(\gamma)\right|: \gamma \in \hat{H}\right\} \\
& =N_{H}\left(\varphi^{*} \mu\right) \text { for all } \mu \in M(G) .
\end{aligned}
$$

Proof. Since $\varphi$ is not open, the induced map $\hat{\varphi}: \hat{H} \rightarrow \hat{G}$ is not proper, so by Proposition 3, for any $\xi \in \operatorname{cl}(\hat{\varphi}(\hat{H})) \subset \hat{G}$, there exists a net $\left\{\gamma_{\alpha}\right\} \subset \hat{H}$ such that $\gamma_{\alpha} \vec{\alpha} \infty$ in $\hat{H}$ and $\hat{\varphi} \gamma_{\alpha} \vec{\alpha} \xi$. Let $\mu \in M(G), \gamma \in \hat{H}$, then

$$
\begin{aligned}
\left(\varphi^{*} \mu\right)^{\wedge}(\gamma) & =\int_{H}(s, \gamma) d \varphi^{*} \mu(s)=\int_{G}(\varphi x, \gamma) d \mu(x) \\
& =\int_{G}(x, \hat{\varphi} \gamma) d \mu(x)=\hat{\mu}(\hat{\varphi} \gamma) .
\end{aligned}
$$

Let $\varepsilon>0$, then there exists $\gamma_{0} \in \hat{H}$ such that $\left|\left(\varphi^{*} \mu\right)^{\wedge}\left(\gamma_{0}\right)\right|>N_{H}\left(\varphi^{*} \mu\right)-\varepsilon$. Now there 
exists a net $\left\{\gamma_{\alpha}\right\} \subset \hat{H}$ such that $\gamma_{\alpha} \rightarrow \infty$ and $\hat{\varphi} \gamma_{\alpha} \vec{\alpha} \hat{\varphi} \gamma_{0}$ so by the continuity of $\hat{\mu}$ (since a Fourier-Stieltjes transform) we have

$$
\begin{aligned}
\lim \sup \left\{\left|\left(\varphi^{*} \mu\right)^{\wedge}(\gamma)\right|: \gamma \rightarrow \infty\right\} & \geqq \lim _{\alpha}\left|\left(\varphi^{*} \mu\right)^{\wedge}\left(\gamma_{\alpha}\right)\right| \\
& =\lim _{\alpha}\left|\hat{\mu}\left(\hat{\varphi} \gamma_{\alpha}\right)\right|=\hat{\mu}\left(\hat{\varphi} \gamma_{0}\right)>N_{H}\left(\varphi^{*} \mu\right)-\varepsilon .
\end{aligned}
$$

This holds for all $\varepsilon>0$.

This yields an easy proof of the following well-known fact.

15. COROLlaRY. If $G$ is a nondiscrete abelian group and $\mu \in M_{d}(G)$ (the discrete measures) then $\mu \notin M_{0}(G)$, in fact lim $\sup \{|\hat{\mu}(\gamma)|: \gamma \rightarrow \infty\}=\sup _{\gamma}|\hat{\mu}(\gamma)|$.

Proof. The identity map $i: G_{d} \rightarrow G$ ( $G_{d}$ denotes $G$ with the discrete topology) is not open, and $i^{*}$ is an isomorphism of $M\left(G_{d}\right)$ onto $M_{d}(G)$.

EXAMPLE. We map $\varphi: R \rightarrow T^{2}$ by $x \mapsto(x, s x)$ where $s$ is irrational and the coordinates are reduced modulo 1 (i.e. $T^{2} \cong R^{2} / Z^{2}$ ). This maps $R$ onto a dense subgroup of $T^{2}$, and is not open.

Rudin [14, p. 88] shows that if $\hat{\varphi}$ is proper then $\varphi^{*}\left(L^{1}(G)\right) \subset L^{1}(H)$. We conjecture that Theorem 14 holds in the case that $H$ is a compact group, and $G$ and $H$ are nonabelian.

We have recently shown for any infinite nondiscrete locally compact group that $M_{d}(G) \cap M_{0}(G)=\{0\}$.

3. Some applications. If $G$ is abelian and $\gamma$ a (continuous) character of $G$ then we define $\gamma^{*}$, a homomorphism of $M(G)$ into $M(T)$ (a character, of course, is a continuous homomorphism of $G$ into $T$, the circle group $\{z \in C:|z|=1\})$. We will study the limiting behavior of $\gamma^{*} \mu$ as $\gamma \rightarrow \infty$ in $\hat{G}$ for a measure $\mu \in M_{0}(G)$. The theorem we prove below depends on the power mappings of $G$ (i.e. $x \mapsto n x$, $n=1,2, \ldots$ ) being open.

16. THEOREM. Let $G$ be a nondiscrete locally compact abelian group with the property that each power map $T_{n}: x \mapsto n x(n=1,2, \ldots$, additive notation) is open, or equivalently by Theorem 8 that each $\hat{T}_{n}: \gamma \mapsto n \gamma$ is proper on $\hat{G}$, and let $f$ be $a$ Riemann-integrable function on $T$ and let $\mu \in M_{0}(G)$. Then

$$
\lim _{\gamma \rightarrow \infty} \int_{T} f d \gamma^{*} \mu=\int_{G} d \mu \int_{T} f d m_{T} .
$$

Proof. Since $M_{0}(G)$ is a band we may assume that $\mu \geqq 0$. As $\gamma \rightarrow \infty, n \gamma \rightarrow \infty$ since $\hat{T}_{n}$ is proper (see Proposition 1). Thus as $\gamma \rightarrow \infty,\left(\gamma^{*} \mu\right)^{\wedge}(n)=\hat{\mu}(n \gamma) \rightarrow 0$ for $n \neq 0$, and $\left(\gamma^{*} \mu\right)^{\wedge}(0)=\hat{\mu}(0)$. Thus as $\gamma \rightarrow \infty,\left(\gamma^{*} \mu\right)^{\wedge} \rightarrow\left(\hat{\mu}(0) m_{T}\right)^{\wedge}$ pointwise on $Z$ $(=\hat{T})$, the integers. Hence as $\gamma \rightarrow \infty, \gamma^{*} \mu \rightarrow \hat{\mu}(0) m_{T}$ in the weak-* topology. The extension to Riemann-integrable functions is standard since $\mu$ was chosen nonnegative (see [9, p. 40]).

REMARK. It is quite easy to prove a converse for this theorem, in fact, if the above limit relation holds just for the function $f\left(e^{i \theta}\right)=e^{i \theta}$ all real $\theta$, then $\mu \in M_{0}(G)$. 
For $G=T$, Theorem 16 shows that if $\mu \in M_{0}(T)$, then as $n \rightarrow \infty$,

$$
\int_{0}^{2 \pi} \chi(n t) d \mu(t) \underset{n}{\longrightarrow} \frac{b-a}{2 \pi} \int_{0}^{2 \pi} d \mu(t)
$$

where $\chi$ is the characteristic function of $(a, b)$. See [12, p. 691] and [16, p. 144] for this case. Thus for $\mu \in M_{0}(T)$, the support of $\mu$ must be distributed over $T$ with a certain degree of homogeneity. It follows that the canonical measure supported on the classical middle third Cantor set is not in $M_{0}(T)[16$, p. 144].

EXAMPLES. In Theorem 16, we assumed that each power map $\hat{T}_{n}: \gamma \mapsto n \gamma$ on $\hat{G}$ is proper. This is the case for $\hat{G}=R$ (the reals), $Z$ (the integers), $\Delta(p)$ (the $p$-adic integers), $p$ prime, $\Omega(p)$ (the $p$-adic numbers), $\sum_{n=1}^{\infty} Z\left(p_{n}\right)$ where $p_{n} \rightarrow \infty$ and $Z(p)$ denotes the finite group with $p$ elements, and finite combinations thereof. The case of $\hat{G}=\sum_{n=1}^{\infty} Z(p)$ for a fixed prime $p$ will be handled in the subsequent proposition.

Suppose $\hat{G}$ is a $\sigma$-compact, locally compact abelian group and $\hat{T}_{n}$ is one-to-one. Now $\hat{T}_{n}$ is proper provided $\hat{T}_{n}(\hat{G})$ is closed in $\hat{G}$ by Corollary 7 . One might conjecture that in any case $\hat{T}_{n}$ will always be proper. That this is not true was communicated to us by Karl Hofmann. Let $\hat{G}$ be the local direct product [7, p. 57] of the 2-adic numbers, $\Omega(2)$, relative to the 2 -adic integers, $\Delta(2)$. Thus $\hat{G}$ consists of elements of the form $\left\{x_{n}\right\}_{n=1}^{\infty} \in \prod_{n=1}^{\infty} \Omega(2)$ where $x_{n} \in \Delta(2)$ except for a finite number of indices. Let $H=\prod_{n=1}^{\infty} \Delta(2) \subset \hat{G}$ be declared to be a compact open subgroup with its product topology. Now $\hat{G}$ is a $\sigma$-compact, locally compact abelian group and $\hat{T}_{2}$ is one-to-one. Now $\hat{T}_{2}$ is not proper since $\hat{T}_{2}^{-1}(H)$ is not compact (equivalently since $\hat{T}_{2}(\hat{G})$ is not closed in $\hat{G}$ ).

17. Proposition. Let $p$ be a fixed prime and let $G=\prod_{n=1}^{\infty} Z(p)$. Let $m_{p}$ be the Haar measure of $\left\{e^{2 \pi i k / p}: k=0,1, \ldots, p-1\right\}$ with total mass 1 , and let $\mu \in M_{0}(G)$. Then as $\gamma \rightarrow \infty$ in $\hat{G}, \gamma^{*} \mu \rightarrow\left(\int_{G} d \mu\right) m_{p}$ in the weak-* topology.

Proof. Use the methods similar to those used in the proof of Theorem 16 .

The fact that $e^{i \theta} \rightarrow e^{n i \theta}, n \geqq 2$, is an ergodic homomorphism of $T$ onto itself and that it is involved with $M_{0}(T)$ by way of the Rajchman theorem leads us to consider ergodic homomorphisms of compact groups in general.

Definition. Let $G$ be a compact (not necessarily abelian) group and let $\varphi$ be a continuous homomorphism: $G \rightarrow G$. We say that $\varphi$ is measure-preserving if $m_{G}\left(\phi^{-1}(E)\right)=m_{G}(E)$ for all Borel sets $E \subset G$. From this one can derive that the induced map $U$ on $L^{2}(G)$ given by $U f(x)=f(\varphi x)$ is an isometry (see [6, p. 13]). Now we say $\varphi$ is ergodic if it is measure-preserving, and $U f=f, f \in L^{2}(G)$ implies $f$ is constant.

REMARK. Rajagopalan and Schreiber [11] have studied ergodic automorphisms. It is easy to see that $\varphi$ is measure-preserving if and only if $\varphi$ is onto $G$ for compact groups. 
18. THEOREM. Let $G$ be compact and infinite, let $\varphi$ be an ergodic homomorphism of $G$ onto $G$, and let $\mu \in M_{0}(G)$, then $\int_{G} f\left(\varphi^{n} x\right) d \mu(x) \rightarrow \int_{G} d \mu \int_{G} f d m_{G}$ as $n \rightarrow \infty$ for all $f \in C(G)$, that is $\left(\varphi^{*}\right)^{n} \mu \rightarrow\left(\int_{G} d \mu\right) m_{G}$ in the weak-* topology as $n \rightarrow \infty$. (This phenomenon is called strong mixing in the case of functions, i.e. if $d \mu(x)=$ $g(x) d m_{G}(x), g \in L^{1}(G)$.)

Proof. We use the notation of [3]. Let $\hat{G}$ be the set of equivalence classes of continuous unitary irreducible representations of $G$, and for each $\alpha \in \hat{G}$ we choose $T_{\alpha} \in \alpha$, so that $T_{\alpha}$ is a continuous homomorphism of $G$ into $U\left(n_{\alpha}\right)$, the group of $n_{\alpha} \times n_{\alpha}$ unitary matrices, where $n_{\alpha}$ is the degree of $\alpha$. For $\alpha \in \hat{G}$ define $\chi_{\alpha}(x)=$ Trace $T_{\alpha}(x)$, the character of $\alpha$. Now if $\varphi$ is an ergodic homomorphism then $T_{\alpha} \circ \varphi$ is again an irreducible representation. This follows from

$$
\int_{G}\left|\operatorname{Trace}\left(T_{\alpha} \circ \varphi\right)(x)\right|^{2} d x=\int_{G}\left|\chi_{\alpha}(\varphi x)\right|^{2} d x=1
$$

Further each set $\left\{\chi_{\alpha} \circ \varphi^{n}: n=1,2, \ldots\right\}$ is infinite, except for $\alpha=0$, the identity representation $G \rightarrow\{1\}$, for suppose that $\chi_{\alpha} \circ \varphi^{n}=\chi_{\alpha} \circ \varphi^{m}$ for some integers $n<m$, then $g=\sum_{j=n}^{m-1} \chi_{\alpha} \circ \varphi^{j}$ would be an invariant function for $\varphi$ and so would be constant, contrary to the orthogonality of distinct (irreducible) characters. This argument is due to Halmos [6, p. 53] in the abelian case, and its applicability to the nonabelian case was pointed out by Kaplansky [8, p. 111].

Now let $\mu \in M_{0}(G), \alpha \neq 0$, then the above indicates that $\left\|\hat{\mu}\left(T_{\alpha} \circ \varphi^{n}\right)\right\| \rightarrow 0$ as $n \rightarrow \infty$ (recall from $\S 2$, the operator norm). Let $f$ be a trigonometric polynomial, that is, $f(x)=c_{0}+\sum_{\alpha \neq 0}$ Trace $\left(C_{\alpha} T_{\alpha}(x)\right)$, where each $C_{\alpha}$ is an $n_{\alpha} \times n_{\alpha}$ complex matrix, and only finitely many $C_{\alpha}$ are nonzero, and $c_{0}=\int_{G} f d m_{G}$. Then

$$
\int_{G} f\left(\varphi^{n} x\right) d \mu(x)=c_{0} \int_{G} d \mu+\sum_{\alpha \neq 0} \operatorname{Trace}\left(C_{\alpha} \hat{\mu}\left(T_{\alpha} \circ \varphi^{n}\right)\right)
$$

which clearly converges to $c_{0} \int_{G} d \mu$ as $n \rightarrow \infty$, using the matrix inequality

$$
|\operatorname{Trace}(A B)| \leqq \operatorname{Trace}\left(A^{*} A\right)^{1 / 2}\|B\| \text {. }
$$

An approximation by trigonometric polynomials to continuous functions (PeterWeyl theorem) finishes the proof.

REMARK. The theorem can be extended to Riemann-integrable functions as in Theorem 16.

EXAmples. Let $n=1,2,3, \ldots$ and consider $T^{n}=R^{n} / Z^{n}$, and let $A$ be an $n \times n$ integer matrix with nonzero determinant having at least one eigenvalue which is not a root of unity. Then $x \mapsto A x$ is an ergodic homomorphism of $T^{n}$. Note that $\hat{A}: Z^{n} \rightarrow Z^{n}$ is given by the transposed matrix and $\left\{\hat{A}^{m} k: m=1,2, \ldots\right\}$ is infinite for each $k \in Z^{n}, k \neq 0$ (see [6, p. 55]). 


\section{BIBLIOGRAPHY}

1. N. Bourbaki, General topology. Part 1, Hermann, Paris; Addison-Wesley, Reading, Mass., 1966. MR 34 \#5044a.

2. C. F. Dunkl, Operators and harmonic analysis on the sphere, Trans. Amer. Math. Soc. 125 (1966), 250-263. MR 34 \#3224.

3. C. Dunkl and D. Ramirez, Topics in harmonic analysis, Appleton-Century-Crofts, New York, 1971.

4. - Translation in measure algebras and the correspondence to Fourier transforms vanishing at infinity, Michigan Math. J. 17 (1970), 311-320.

5. P. Eymard, L'algèbre de Fourier d'un groupe localement compact, Bull. Soc. Math. France 92 (1964), 181-236. MR 37 \#4208.

6. P. Halmos, Lectures on ergodic theory, Publ. Math. Soc. Japan, no. 3, Math. Soc. Japan, Tokyo, 1956; reprint, Chelsea, New York, 1960. MR 20 \#3958.

7. E. Hewitt and K. A. Ross, Abstract harmonic analysis. vol. 1: Structure of topological groups. Integration theory, group representations, Die Grundlehren der math. Wissenschaften, Band 115, Academic Press, New York: Springer-Verlag, Berlin, 1963. MR 28 \#158.

8. I. Kaplansky, Groups with representations of bounded degree, Canad. J. Math. 1 (1949), 105-112. MR 10, 428.

9. K. R. Parthasarathy, Probability measures on metric spaces, Academic Press, New York, 1967. MR 37 \#2271.

10. L. S. Pontrjagin, Continuous groups, GITTL, Moscow, 1954; English transl., Gordon and Breach, New York, 1966. MR 17, 171; MR 34 \#1439.

11. M. Rajagopalan and B. Schreiber, Ergodic properties of automorphisms of a locally compact group. II (to appear).

12. A. Rajchman, Une classe de séries trigonométriques qui convergent presque partout vers zéro, Math. Ann. 101 (1929), 686-700.

13. W. Rudin, Measure algebras on abelian groups, Bull. Amer. Math. Soc. 65 (1959), 227-247. MR 21 \#7404.

14. - Fourier analysis on groups, Interscience Tracts in Pure and Appl. Math., no. 12, Interscience, New York, 1962. MR 27 \#2808.

15. A. Weil, L'intégration dans les groupes topologiques et ses applications, 2nd ed., Hermann, Paris, 1951.

16. A. Zygmund, Trigonometric series. Vol. 2, 2nd ed., Cambridge Univ. Press, New York, 1959. MR 21 \#6498.

UNIVERSITY OF VIRGINIA,

Charlottesville, Virginia 22901 